\title{
“Authors' Reply: Emerging Evidence on Multisystem Inflammatory Syndrome in Children Associated with SARS-CoV-2 Infection"
}

\author{
Mangla Sood $^{1}$ (D) - Seema Sharma ${ }^{2}$ \\ Accepted: 25 June 2021 / Published online: 8 July 2021 \\ (C) The Author(s), under exclusive licence to Springer Nature Switzerland AG 2021
}

\begin{abstract}
With increased patients of severe acute respiratory syndrome coronavirus 2 infection, more children with multisystem inflammatory syndrome (MIS-C) are being reported worldwide. Our focus is to share the new evidence-based findings and experiences during the early stage of the pandemic with the researchers on a larger platform. We hope this review will help to frame appropriate strategies and guidelines for management of MIS-C.
\end{abstract}

Keywords Multisystem inflammatory syndrome in children (MIS-C) · SARS-CoV-2

We thank the reader for reading our review with interest [1]. This review was undertaken and reported in accordance with the preferred reporting items for systematic review and metaanalyses (PRISMA) guidelines [2]. The details have been shared in the supplementary material [1].

Systematic reviews assessing the effectiveness of an intervention or therapy use the PICO approach. Our systematic review was not intended to "summarize the findings of several clinical trials that evaluate the effectiveness or efficacy of a similar treatment approach on similar group of patients". In the present systematic review, we intend to review and summarize the available evidence on MIS-C to synthesize the overall prognosis based upon expert opinion. We focussed on paediatric population using the words infant, newborn, child, and adolescent as has been mentioned in search strategy. Detail of contribution by each author were shared while submission of the paper.

This article is part of the Topical Collection on Covid-19

Mangla Sood

drmanglasood@gmail.com

Seema Sharma

dr.seema73.ss@gmail.com

1 Department of Pediatrics, Indira Gandhi Medical College, Shimla, Himachal Pradesh 171001, India

2 Department of Pediatrics, Dr RP Govt. Medical College, at Kangra, Tanda, India
MEDLINE is a great resource for medical research, and PubMed is a free interface to search primarily the MEDLINE database of references and abstracts on life sciences and biomedical topics. Same has been done by us. Databases like EMBASE are paid not accessible through our institution, hence not used for searching papers. The data was extracted on excel sheet which was used for descriptive stats, and meta analysis. All included studies were published and peer reviewed, as can be verified from the references. The extracted data was properly reviewed by all authors.

There are several tools for assessing the methodological quality of observational studies. One among them is the STROBE checklist which was used by us for evaluating the reporting of observational studies [3].

In case reports and observational studies, population characteristics and exposure or outcome definitions will likely differ across studies, hence heterogeneity. Inclusion of case series is not uncommon in systematic reviews; it could be seen as a cohort study without a control group. A meta-analysis thoroughly examines a number of valid studies on a topic and mathematically combine the results using accepted statistical methodology to report the results as if it were one large study.

As the COVID is an evolving disease and new facts and research is still on its way. We acknowledge that during this challenging emergency times, scientific communication should be promoted; the focus is to share the new findings and experiences during the early stage of the pandemic with the researchers on a larger platform so that appropriate strategies can be evolved timely to control the spread of this virus. 


\section{References}

1. Sood M, Sharma S, Sood I, Sharma K, Kaushik A. Emerging evidence on multisystem inflammatory syndrome in children associated with SARS-CoV-2 infection: a systematic review with meta-analysis. SN Compr Clin Med. 2021;3(1):38-47.

2. Page MJ, McKenzie JE, Bossuyt PM, Boutron I, Hoffmann TC, Mulrow $\mathrm{CD}$, et al. statement: an updated guideline for reporting systematic reviews. BMJ. 2021;2020:372-80.
3. STROBE Statement: Available checklists [Internet]. [cited 2021 Jun 12];Available from: https://www.strobe-statement.org/ index.php?id=available-checklists

Publisher's Note Springer Nature remains neutral with regard to jurisdictional claims in published maps and institutional affiliations. 